\title{
Two electrodes system: performance on ECG, FECG and EMG detection
}

\begin{abstract}
Recording of biopotential signals is uneasy as interference and noise can come from the preparation of the subject, environment setting and other biological signal. The circuit design in acquisition system must good and meets specification as is not distort original signal and safe to patient. Classic design of biosignal acquisition system usually use third electrode as reference potential. The third electrode is not picking up the biological signal and placed quite far from the measured signal as it can help to remove common mode signal. However, sometime the third electrode will limit patient movement since some of the experiment requires the patient or subject do several kind of movement during signals recording. Therefore, two electrodes system without third electrode may provide mobility and comfort since number of electrode patched on the body is reduced. Though several previous researches have implemented the two electrodes system, but they only tested on electrocardiogram. Hence, the main contribution of this paper is investigating the ability and performance of the proposed system on fetal electrocardiogram and electromyogram detection since the challenges to detect both signals are different compare to electrocardiogram. The electrocardiogram, fetal electrocardiogram and electromyogram are successfully detected using the propose system but with several filtering stages. The performance evaluation include signal to noise ratio after the filtering stage and accuracy of the signal detection.
\end{abstract}

Keyword: Electrocardiogram; Fetal electrocardiogram; Electromyography 